\title{
Construction and Analysis of the Decision Model based on the Transfer of Human Resources
}

\author{
Xu Ting \\ Shunde Polytechnic (china) \\ 29770041@qq.com
}

\begin{abstract}
The transfer of human resources refers to the transfer of human resources across different regions, industries and organizations depending on the development trends in the respective areas, economic environment and personal development needs It is a process where the revenue of human resources improves continuously, and regional economy, industries and organizations are growing. Reasonable transfers across regions, industries and organizations can further realize the potential of individuals and enhance their advantageous position in the job market. Moreover, the transfers can optimize human resource allocation and the overall regional industrial development, improving the utilization of human resources. According to the law of diminishing returns, when there is perfect competition across regions, industries and orgonizations, the marginal utility of the side that has human resources shortage is hoher than the side that has surplus. The human resources will flow to the side with shortage till the marginal revenue of the two sides reaches equilibrium. This is the ideal condition for human resources transfer. This paper is aimed to build models fôr indindual human resource decisionmaking, organizational decision-moking and dynamic balance respectively, in order to facilitate the equilibrium transfer ff human resources across different regions, industries and organizations, and finally ta achieve the optmal allocation of human resources.
\end{abstract}

Keywords: the transfer of human resources; decision-making model

\section{Introduction}

The transfer of human resources refers to the transfer of human resources across different regions, industries and organizations depending on the development trends in the respective areas, economie environment and personal development needs. It is a process where the revenue oChuman resources improves continuously, and regional economy, industries and organizations are growing (Gregory, 2012). Reasonable transfers across regions, industries and organizations can further realize the potential of individuals and enhance their advantageous position in the job market. Moreover, the transfers can optimize human resource allocation and the overall regional industrial development, improving the utilization of human resources. The Figure below shows how reasonable human resources transfer results in a win-win situation. 


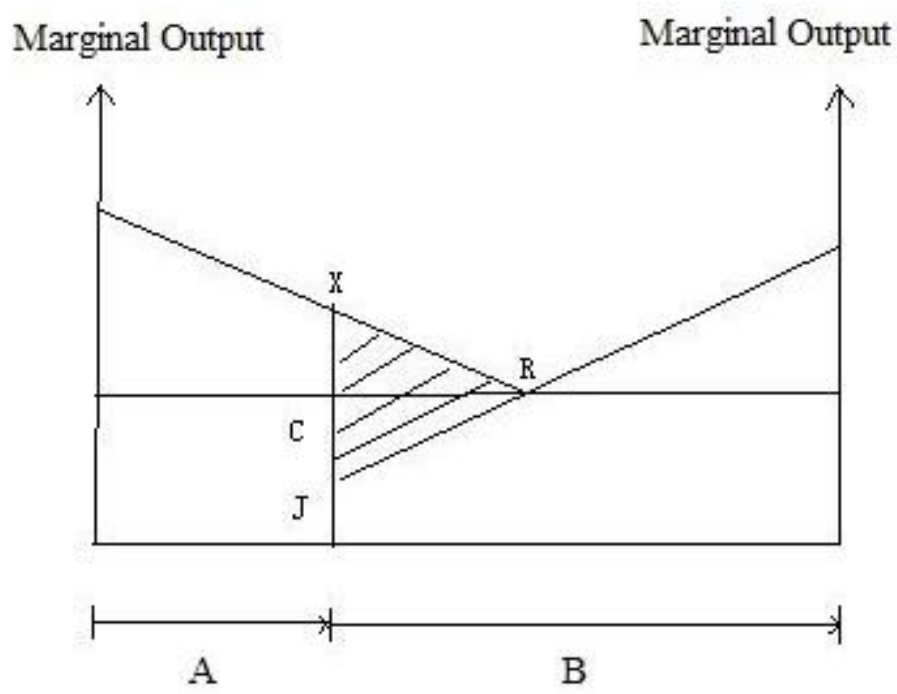

In the Figure, A is short in human resources, while B has surplus in human resources. According to the marginal revenue theory of human resources, A has higher returns of human resources revenue than $\mathrm{B}$. As a result, if balanced and reasonable transfer takes place, human resources will move from B to A till the two sides' marginal revenues achieve balance (in $\mathrm{R}$ point). For side $\mathrm{A}$, although the marginal revenue of human resources decreases, the balanced and reasonable transfer eases its resource shortage, and brings benefits in terms of advanced think ng, technology innovation, and capital. In the Figure, the additional returns are represented by CXR for B, although there is outflow of surplus and redundant human resources, its marginal revenue increases, which solves the problem of human resources reduncaney and waste, and enhances the efficiency of using capital, equipment, venues and ther resources In the Figure, the additional returns of B are represented by CJR. From the above, balanced and reasonable human resources transfer can bring additional revenues to $\mathrm{A}$ and $\mathrm{B}$, and achieve equilibrium between the marginal revenues. Thas the two sides gain a win-win situation (the additional revenues for them are represented by JXR)

\section{Review 2}

\subsection{The Main Problems of the Transfer of Human Resources at Present}

\subsubsection{The Imbalance and Unipolarity of the Transfer of Human Resources Has Led To Uneven Allocation of Human Resources}

The balanced transfer of human resources is conducive to optimizing the HR structure withim organizations, industries, and regions, but this positive effect is based on the dynamic equilibrium of the transfer. That is to say there is a movement of human resources, the surplus and unnecessary personnel will flow out, while the personnel in shortage will flow in, such movement contributes to the dynamic equilibrium of human resource allocation across organizations, industries, regions (Alin and Smeds, 2011). At present, the trend of the transfer is usually appeared as from less developed areas move to developed areas, from less robust organizations and industries move to the one with promising prospects and higher pay. This one-way transfer of human resources has led to an imbalance of human resources allocation. 


\subsubsection{The Frequent and High-Level Transfer of Human Resources Has Led to the Instability of Human Resources Allocation}

With the rapid development of the modern market economy, competitions in key areas, industries, organizations change as well. The traditional competition is the competition of products, however nowadays the competition across regions, industries and organizations will leverage on human resources, science and technology to gain advantage. As the hinder of the transfer gradually reduced, the frequency of human resource transfer was significantly accelerated (Kenneth, 2014). In the process of human resources contention, senior management personnel and technical talents with rich human resources experience and high technical level, are more likely to generate greater benefits for the region, industry and organization they are in. As a result, this group of people become the main target of contention in talent's market, which has led to higher mobility. Such frequent and high-rise transfer of human resources brought uncertainties to the backbone personnel in the region, industry and organization, which is difficult to maintain a relatively stable human resources allocation.

\subsubsection{No Sound Laws and Regulations To Standardize the Transfer of Human Resources}

At the national level, currently there is no laws passed through the Kegislation of the National People's Congress that to regulate the transfer of human resources. While there are some local regulations on human resource transfer, yet they are neither authoritative nor complete, so it quite difficult to regulate the transfer of luman resources (Peng, 2014). On the one hand, there are obvious signs of a planned economy in the current laws and regulations. As there is no personnel arbitration body, is independent of government personnel departments, thus it will influence lavenforeement efforts; on the other hand, existing laws and regulations appry light punishment on organization or individual's breach, therefore the cost of breach is quite low

\subsection{Domestic and International Research Issues and Problems on the Transfer of Human Resources}

Among the earliest group of foreign scholars who studied on the phenomenon of human resource transfers, British statisticians Ernest-George Ravenstein is one of the most important researchers (1n this field. In his book The Laws of Migration, he proposed that discrimination, oppression, and dissatisfaction of material life or spiritual life are the reason that drive the ctansfer of human resources, among which the economic factor is the major reason (Laux and Leuz, 2009). US scientists Lewin developed the function relationship between the individual and the organization's environment, he considered various factors that the change of environment may lead to the transfer of employees.

\section{$B=f(p \cdot e)$}

In this equation, $B$ is individual performance, $P$ is individual ability, $e$ is the envifonnent. The equation reveals that individual ability and the environment will directly affects the individual's work performance. There is a similar field of physics relationship exists between individual performance, individual ability and the environment, which is to say, the individual performance is not only relate to one's capacity and quality, but also closely relate to the environment.

R. Herberle in The Causes of Rural-urban Migration pointed out that migration is not caused by a single factor, it is the result of many factors combined, including the push and pull. After a series of studies, he developed the "push - pull" theoretical model, which laid the foundation for future in-depth study on the transfer of human resources (Frederick, 2013).

American scientist Katz study on the life of organizations, he found that the length of the life of an organization is related to the communication status within the organization. 
He believes that members of the organization usually will have better communication performance within the first 1-5 years, and therefore will achieve more fruitful results; however, if members in the organization have stayed together for more than five years, they are likely more familiar with each other and communicate less than before, therefore the thinking stereotype will lead to cognitive assimilation, then he organization will be aging and losing its vitality. The solution is to optimize human resources in organizations through the continuous transfer of human resources (Plantin, 2009).

British economist David Ricardo clarifies the relationship between the developed and underdeveloped industry. In his On the Principles of Political Economy and Taxation, he proposed two basic hypotheses: First, underdeveloped industry showed a trend of diminishing returns, that is, the investment maintains unchanged, but the output will be declining; Second, even if developed industry accepted the surplus human resources from underdeveloped industry, it will not lead to a rise in overall wages. This two basic hypotheses reflect the necessity and possibility of the transfer of human resources (Farndale, 2010).

Professor Li Baoyuan from Beijing Normal University, pointed out in his monograph Human Capital and Economic Development, that the transfer of human resources market is the inherent natural requirements of a market economy. The tansfer of human resources is achieved by investing a certain amount of capital, and it is an economic behavior achieved by the capital investment. The decision is made through the comparison between the benefit and cost of the transfer of human resources (He, 2013).

There are some scholars believe that human resources is a special form of resources of production factors, thus the transfer of human esources is within the scope of the transfer of production factors. For any region/industry/organization human resources are a key factor for sustainable development, the wealth created by the transfer of human resources can be redistribute across different regions, industries, and organizations for mutual benefit. From the perspective of optimal allocation of resources and the perspective of sustainable development, the transfer of humaresources is efficient (Gregory, 2012).

The theories above demonstrate the necessity of the transfer of human resources from different angles. Some of chem are from growth, stimulate creativity angles to study the transfer of human resoarces, some are from pure economics angle, to study the decisionmaking of the transfer by comparing the cost and benefits of the transfer; and some of the theories are fromamechanism angle that market and administrative mechanisms will lead the transfer of human resources. However, these theories failed to provide detailed analysis on the factors and variables that influence the transfer of human resources, and the relationship between the variables and their mutual influence, and yet they haven't studied the transfer of human resources and built decision-making model based on multivariate observation analysis.

\section{Construetion and Analysis of Decision Model Based on the Transfer of Human Resources \\ 31. Factors Influencing the Transfer of Human Resources \\ Based on the study of the decision model of the transfer of human resources, Professor} Feng Zibiao in Shanxi University of Finance and Economics elaborates on the formation, transfer and allocation of human resources based on the operation of human resources. Using the cost - benefits analysis in economics, he constructs a decision model of the transfer of human resources. In the model, he reduces the various factors of transfer of human resources to two elements: the cost of transfer and the benefit of transfer. Through comparing the cost and benefit, the transfer of human resources is analyzed. 


$$
S=\sum_{\mathrm{t}=1}^{\mathrm{n}} \frac{M O_{t}}{(1+r)^{t}}-\sum_{t=1}^{n} \frac{I_{t}}{(1+r)^{t}}=\sum_{t=1}^{n} \frac{M O_{t}-I_{t}}{(1+r)^{t}}
$$

In the model, $\mathrm{S}$ stands for net value of benefit, $\mathrm{r}$ stands for annual interest rate, $\mathrm{t}$ stands for year, $\mathrm{MO}_{\mathrm{t}}$ stands for the difference of value between the revenue in the year $\mathrm{t}$ and the revenue in the original job, $\mathrm{I}_{\mathrm{t}}$ stands for the direct and indirect currency cost incurred from the transfer in the year $t$.

This model explains that the decision of human resources transfer is based on the amount of the net benefit. However, this model only takes into account few factors such as the year, and the interest rate. In addition to the factors including year, interest rate, this paper takes broader variables into account more comprehensively, including coefficient of environmental barriers, coefficient of age, required rate of return, marginal revenue of human resources. This paper is aimed to study the transfer of human resources and construct the decision model based on the observation and analysis of multiple variables.

\subsection{Construction and Analysis of Individual Decision Making Model}

Human resources has its distinctive features - it is the only factor with subjective initiative among all the other productivity factor. The subjective initiative of human resources is a reflection of human being's pursuit of natural value. Human resources have thought, emotion, will and personality, these self-awareness of human resources as an internal force to push the subjective initiative and drive he independent movement behavior of human resources. Different regions, industries, organizations will affect the values, ways of thinking, behavior and other aspects of human resources all the time (Katrin, 2010). Human resources vill be selected according to geography, religion, culture, wealth, values and other factors. As long as there is a gap between regions/industries/organizations, there will be new desire and requirements for human resources. New changes in the environment will lead to the transfer of human resources which is a basic form to achieve human resource reallocation. The following model is able to analyze the individual decisions of human resource.

\section{$\mathbf{P}=\mathbf{I} \times \mathbf{E}$}

In the formula (1), $P$ stands for the expected return, $I$ stands for the transfer of added value, namely the added transfer value of the expected benefits from point A to point $B$, which including the improvement of salary, working environment, and individual status. $I$ represents an individual's strength of transfer motivation. $E$ represents an individual's expected value in terns of their analysis and judgement of point B. Whether to have the expected salary and benefits, promising prospects for the development, as well as the level of risk are all important factors. If an individual believes that transferring to point $B$ will have lower probability to achieve the expected development, even $I$ is high, the expected earning will still be low due to the lower $E$; or if I itself is low, and in the mean time $E$ is not high, the individual will choose to stay at point A. However, on the other hand if $I$ is not high, but due to $E$ has larger value to drive the expected earning higher, or $I$ itself is high, and at the same time $E$ is high as well, then then the individual will choose to transfer to point B. In this formula, $E$ play a leading role.

$$
I=\sum_{m=1}^{n}\left[\frac{\left(Y_{b m}-Y_{a m}\right) \cdot q_{2}}{(1+h)^{m} \cdot q_{1}}+\left(S_{b m}-S_{a m}\right)\right]-\left(L_{1}+L_{2}\right)
$$

In the formula (2), $Y_{b m}$ stands for the direct earnings from point $\mathrm{B}$ in $m$ years, $Y_{a m}$ stands for the direct earnings from point $\mathrm{A}$ in $m$ years, $q_{1}$ stands for the coefficient of 
environmental barriers, $q_{2}$ stands for the coefficient of age, $h$ stands for the necessary rate of earning, $\mathrm{S}_{\mathrm{bm}}$ stands for the indirect earning from point B in $m$ years, $\mathrm{S}_{\mathrm{am}}$ stands for the indirect earning from point $A$ in $m$ years, $L_{1}$ stands for the transfer of direct cost, and $\mathrm{L}_{2}$ stands for the transfer of indirect cost.

$$
K=\frac{T}{T_{b \max }}=\frac{I \times E}{T_{b \max }}
$$

In the formula (3), $K$ stands for the relative utility of the net gain after transfer to point $\mathrm{B}, T_{\text {bmax }}$ stands for the highest satisfaction at point $\mathrm{B}$.

Combine formula (1), (2), (3), we have the decision-making model for the individual transfer of human resources:

$$
K=\frac{\sum_{m=1}^{n}\left[\frac{\left(Y_{b m}-Y_{a m}\right) \cdot q_{2}}{(1+h)^{m} \cdot q_{1}}+\left(S_{b m}-S_{a m}\right)\right] \cdot E-\left(L_{1}+L_{2}\right)}{T_{b \max }}
$$

As can be seen from the model, only when $K$ is getting larger, that is only when an individual human resource transferred to point $\mathrm{B}$, the relative utility of net gain is greater than the one at point $\mathrm{A}$, the individual human esource will Gake the transfer.

\subsection{Construction and Analysis of Organizational Decision-making Model}

In addition to the individual decision-making, the transfer of individual human resource also depends on the organization's decision making. According to the human capital property theory, the organizations investment in human capital emphasizes the cost and the investment return. An organization will consider how much profit will the investment bring, and how much hum capital effectiveness will gain. It emphasizes on the added value that brought by human resources portfolio, and the speed and range of the value (Kim, 2011). According to the social attributes of human capital property theory, the organization will invest on human resources individual growth and development, thus the capital property of of indevidual human resources should belong to the organization as well. Every organization will make its decision based on its own development needs and human capital investment return. The following model can use to analyze the organization's decision making.

(1)Part A : Humąn Resources Deficient

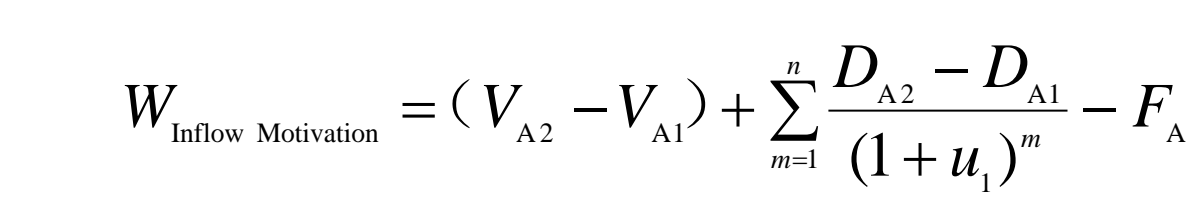

In the formula, $V_{A 2}$ stands for the human resource marginal revenue of Part $\mathrm{A}$ after the inflow of human resources; $V_{A l}$ stands for the human resource marginal revenue of Part $\mathrm{A}$ before the inflow of human resources. With the continuous inflow of human resources, the human resources marginal revenue of Party A will gradually decrease; however with the gradual inflow of human resources, it will bring benefits for Party A in terms of new concepts, technologies and so on. $D_{A 2}$ stands for the benefits of concepts and technologies that brought by the inflow of human resources; $D_{A l}$ stands for the benefits of concepts and technologies that before the inflow of human resources; $F_{A}$ stands for Party A's cost of recruitment and resettlement of manpower; and $u_{l}$ stands for Party A's require rate of return. 
(2)Part B: Human Resources Surplus

$$
W_{\text {Outflow Motivation }}=\left(V_{\mathrm{B} 2}-V_{\mathrm{B} 1}\right)+\sum_{m=1}^{n} \frac{G_{\mathrm{B} 2}-G_{\mathrm{B} 1}}{\left(1+u_{2}\right)^{m}}-F_{\mathrm{B}}
$$

In the formula, $V_{B 2}$ stands for the human resource marginal revenue of Part B after the outflow of human resources; $V_{B I}$ stands for the human resource marginal revenue of Part A before the outflow of human resources. With the continuous outflow of human resources, the human resources marginal revenue of Party B will gradually increase. $G_{B 2}$ stands for Party B's revenue in terms of equipment, funds, space utilization efficiency, etc after the outflow of human resources; $G_{B I}$ stands for Party B's revenue before the outflow of human resources. With the continuous outflow of the surplus human resources, Party B's revenue will gradually increase. $F_{B}$ stands for the Party B's cost of salary and benefits, trainings before the outflow; and $u_{2}$ stands for Party B's require rate of return.

(3)

$$
\mathrm{W}_{\text {Inflow Motivation }}=\mathrm{W}_{\text {Outflow Motivation }}
$$

At this moment, the human resources marginal revenue of Party A and B reach balance, both sides can get additional income and achieve a win-win situration.

\subsection{Construction and Analysis of Dynamic Equilibrium Model}

$$
\begin{aligned}
& \mathrm{W}_{\text {Inflow Motivation }}=\mathrm{W}_{\text {Outflow Motivation }}\left[\frac{\left(Y_{b m}-Y_{a m}\right) \cdot q_{2}}{(1+h)^{m} \cdot g_{1}}+\left(S_{b m}-S_{a m}\right)\right]>\sum_{m=1}^{n}\left(L_{1}+L_{2}\right)
\end{aligned}
$$

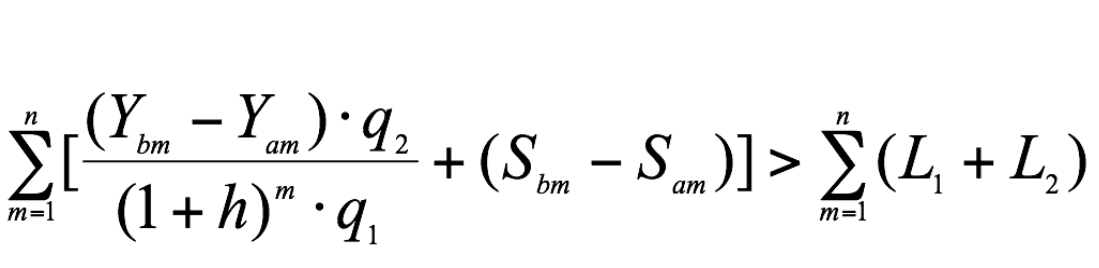

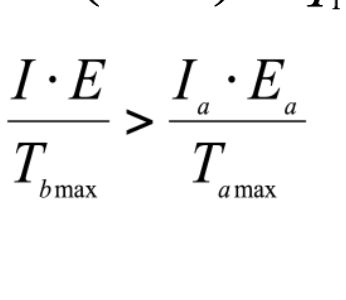

Combine fomula (1), (2), (3) we can draw the conclusion: from an individual's perspective, when individual human resources is expected to transfer to a new organization, the expected benefits is greater than the necessary costs of the transfer; while if the individual's expected net income at the new organization is greater than the original organization, then th individual will make the transfer; from an organizational perspective, when the human resources outflows from organizaitons with surplus and idle human esources, the marginal revenue of organizaitons with deficient human resources will reduce at first, however with the gradual inflow of human resources, organizaitons with deficient human resources will gain great benefits in terms of advanced concepts and methods, technological innovation, capital etc. For the organizaitons with surplus and idle human resources, with the gradual outflow of surplus human resources, the marginal revenue will gradually increase. And at the same time, the utilization efficiency of equipment, funds, space and other resources will be improved, which can effectively solve the idle and waste issues of human resources. 


\section{Case Study}

Take Foshan tourism industry as an example, Foshan tourism industry has low investment, high return, correlation, and other characteristics. Now as the tourism industry is at a rapid growth, high change period, there is much room for development, which led to human resources in some of the traditional, less advanced industry gradually transferred to the tourism industry. At the same time, the current highly competitive tourism industry, to a certain extent, has also contributed to the high-speed transfer of tourism industry employees. According to the industry reports and randomly sampling survey issued by Foshan City Tourist Association, we present sample statistics of Foshan tourism industry employees as follows (Table 1):

\section{Table 1.Sample Statistics of Foshan Tourism Industry Employees}

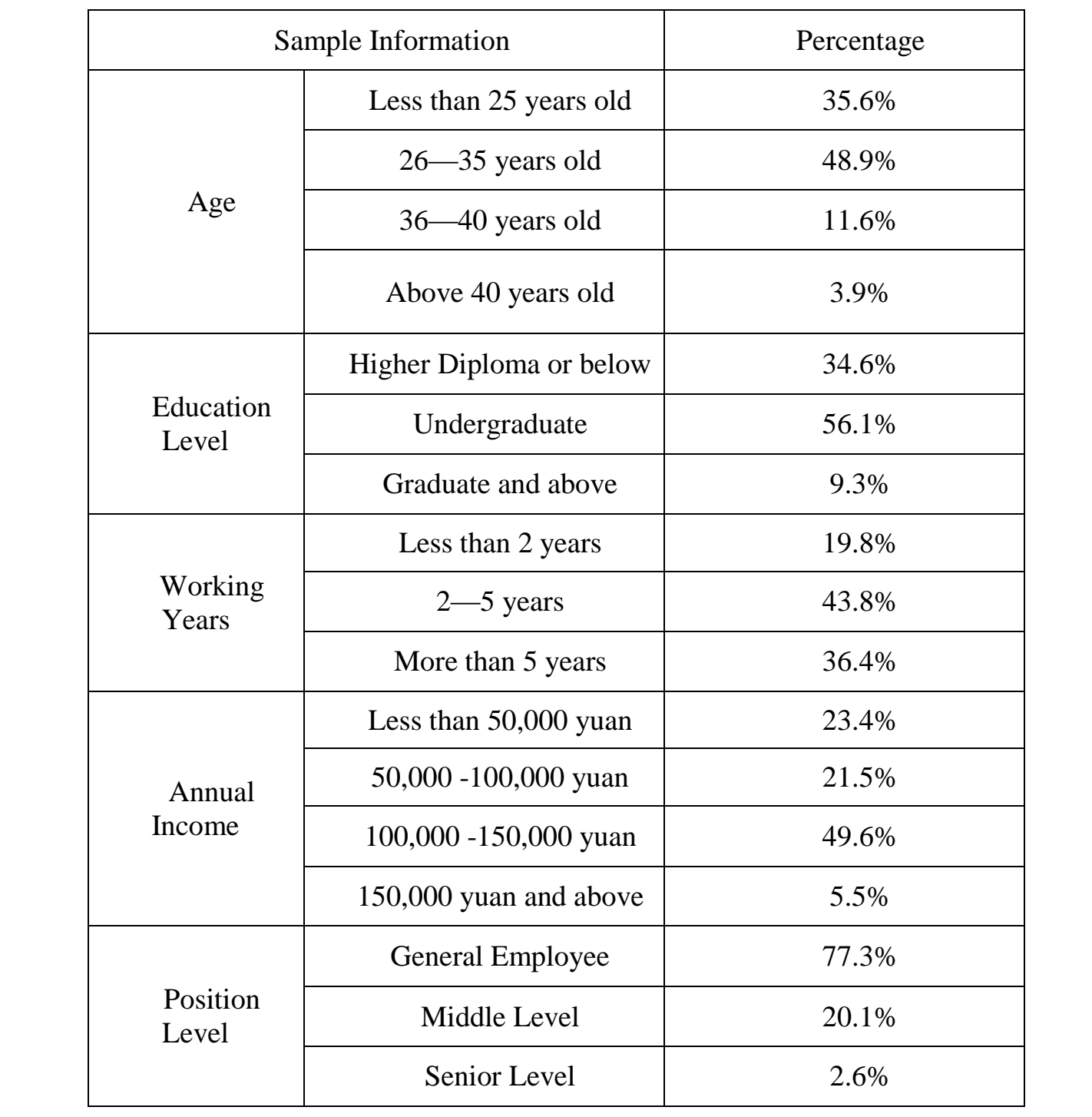

Based on the statistical characteristics of sample employees in Foshan tourism industry, we analyze the transfer status of the employees. (Table 2) 


\section{Table 2. Human Resources Transfer Status of Foshan Tourism Industry Employees1}

\begin{tabular}{|c|c|c|}
\hline \multicolumn{2}{|c|}{ Working Organization } & Job-hopping Percentage \\
\hline \multirow{2}{*}{ Hotel } & $\begin{array}{c}\text { 4-stars and above } \\
\text { (including 4 stars) }\end{array}$ & $73.6 \%$ \\
\cline { 2 - 3 } & Below 4-stars & $51.3 \%$ \\
\hline \multirow{2}{*}{$\begin{array}{c}\text { Travel } \\
\text { Agency }\end{array}$} & Large & $67.8 \%$ \\
\cline { 2 - 3 } Tourist & Small and medium sized & $38.3 \%$ \\
\cline { 2 - 3 } attractions & others & $35.2 \%$ \\
\hline
\end{tabular}

According to the statistical characteristics of samples obtained, and combined with the human resource transfer decision-making model constructed as well as Foshan local environmental factors, the industry average salary, price index, geographic location, etc., with coefficient of environmental barriers and coefficient of age, finally we can get the following conclusions:

In Foshan, human resources from sometraditional industries are transferred to the tourism industry because of the high eanings, quick return and low barriers of entry of the tourism industry. That is, coefficient of human resources individual barriers is low, the earnings of transfer are significantly highen than the costs, and the value-added is significantly higher than the former industry after human resources individual transferred to the tourism industry. Therefore, human resources are willing to transfer to the tourism industry.

The proportion of people who had job-hopping experience among employees of luxury hotels and large travel agencies is significantly higher than small and medium sized hotels and travel agencies. Because a large part of employees of luxury hotels and large travel agencies came from smaland medium sized hotels and travel agencies; but many employees of small an medium sized hotels and travel agencies are fresh graduates or transferred from other industries.

Many executives and middle managers at small and medium sized hotels and travel agencies generally have working experience in luxury hotels and large travel agencies. As the tourism Industry employees are constantly transferred to luxury hotels and large travel agencies, it leâds to large surplus of human resources in luxury hotels and large travel agencies, therefore their career development will be limited. They will get greater development potencial thanks to their rich experiences and customer resources after they trans ferred from luxury hotels and large travel agencies to small and medium sized hotels and travel agencies.

Tourist attractions are less attractive for tourism industry employees due to low economic benefits. There is a lower proportion of those who had job-hopping experience, because few tourism industry employees are willing to transfer to tourist attractions. Currently employees in Foshan tourist attractions mainly include older employees, young people with lower education level, and temporary staff. The transfer of human resources in this area is less active.

The transfer of human resources between luxury hotels, large travel agencies and small and medium sized hotels, travel agencies is better, both sides get the added benefits, and 
that promote the development of Foshan tourism industry; yet the transfer status in tourist attractions is not ideal. Further research on how to improve the situation is needed.

\section{Conclusion}

The decisions of individuals and organizations are complicated and are formed under the influence of multiple factors and variables which are subject to various conditions. The process is like a huge and complex multi-dimensional system that regulates and controls the behaviors of individuals and organizations (Parry, 2011). Traditional analysis based on one variable or two variables usually entails loss of useful information and introduction of useless information in the selecting and processing stages. This will lead to difficulties in distinguishing between primary and secondary issues or even reaching improper or wrong conclusion (Truss, 2009). The paper analyzes factors and variables that influence the decisions of organizations and individual human resource on making, transfers such as the cost of transfer, estimated returns, appreciation of value, coefficient of environmental barriers, coefficient of age, required rate of return, marginal revenue of human resource. Based on these universally applicable factors, it constructs the decision models of individual human resource, organizations and dynamic equilibrium and provides scientific basis for the decisions of transfer for indivdduals and organizations. It serves as an important guarantee for improving the revenue of utilizing human resource and optimizing the distributional structure of hum an resources across regions, industries and organizations.

It can be assumed that in a perfect management and decision mechanism of equilibrium transfer of human resources, and under the joint influence of government macro-control, market allocation, self-initiated adjustment of regions, industries and organizations and free choices of individuals based on rationality, the balanced transfer of human resources can realize optimal allocation of human resources in job positions and reasonable integration of regions, industries and organizations. In consequence, the value of the individual human resource will be tapped adequately while the structure of human resource and allocation of resources will be optimized and the revenues (in economic development, knowledge innovation and inheritance, product innovation and selfdevelopment) will graddually reach the maximum.

\section{Acknowledgements}

2013 Guangdong Province Educational Science "12th 5-year Plan” Research Project, Project No. 2013JK304

2011 Guangdorg Province Vocational Education Teaching Reform Project, Project No. GZYB2011038

2013 Foshan City Philosophy and Social Science Planning Project, Project No. 2013WJ13

\section{References}

[1]. Alin and R. Smeds, "Knowledge transformation in project networks; A speech act level crossboundary analysis", Project Management Journa1, vol. 42, no. 4, (2011), pp. 58-75.

[2] E. Farndale, "Context-bound configurations of corporate HR functions in multinational corporations", Human Resource Ma-nagement, vol. 49, no. 1, (2010), pp. 45-66.

[3] P. Frederick, "Extending Corporate Social Responsibility Research to the Human Resource Management and Organizational Behavior Domains: A Look to the Future. Personnel Psychology", vol. 23, no. 4, (2013), pp. 126-141.

[4] A. Gregory, "Leadership, Innovation Climate, and Attitudes Toward Evidence-Based Practice During a Statewide Imple Verdanamentation", Journal of the American Academy of Child \& Adolescent Psychiatry, vol. 22, no. 1, (2012), pp. 352-364. 
[5] A. Gregory, "Expanding the Domains of Attitudes Towards Evidence-Based Practice: The Evidence Based Practice Attitude Scale-50", Administration and Policy in Mental Health and Mental Health Services Research, vol. 35, no. 5, (2012), pp. 602-618.

[6] F.N. He, "Analysis of human resource allocation by factor analysis", Enterprise Economy, vol. 6, (2013), pp. 63-66.

[7] A. Katrin, "Organizational citizenship behaviours in relation to job status, job insecurity, organizational commitment and identification, job satisfaction and work values", Journal of Occupational and Organizational Psychology, vol. 26, no. 1, (2010), pp. 768-784.

[8] K. De RoeckUnderstanding employees' responses to corporate social responsibility: mediating roles of overall justice and organisational identification. The International Journal of Human Resource Management, 53 (1),..(2014). 30-45.

[9] S. Kim, "Social capital of the HR department, HR's change agent role, and HR effectiveness: Evidence from South Korean", International Journal of Human Resource Management, vol. 22, no. 8, (2011), pp. $1638-1653$.

[10] C. Laux and C. Leuz, "The crisis of fair value accounting: making sense of the recent debate", Accounting, Organization and Society, vol. 34, no. 6-7, (2009), pp. 826-834.

[11] E. Parry, "An examination of E-HRM as a means to increase the value of the HR function", International Journal of Human Resource Management, vol. 22, no. 5, (2011), pp. 1146-1162.

[12] Z. Peng, "Research on human resource decision making based on ILM analysis", Enterprise Management, vol. 5, (2014), pp. 117-118

[13] J. Plantin, "Learning by holding and liquidity", Review of Economics Studies, vol. 76, ho. 1) (2009), pp. 395-412.

[14] C. Truss, "Changing HR functional forms in the UK public sector", Internationa Journal of Human Resource Management, vol. 20, no. 4, (2009), pp. 717-737.

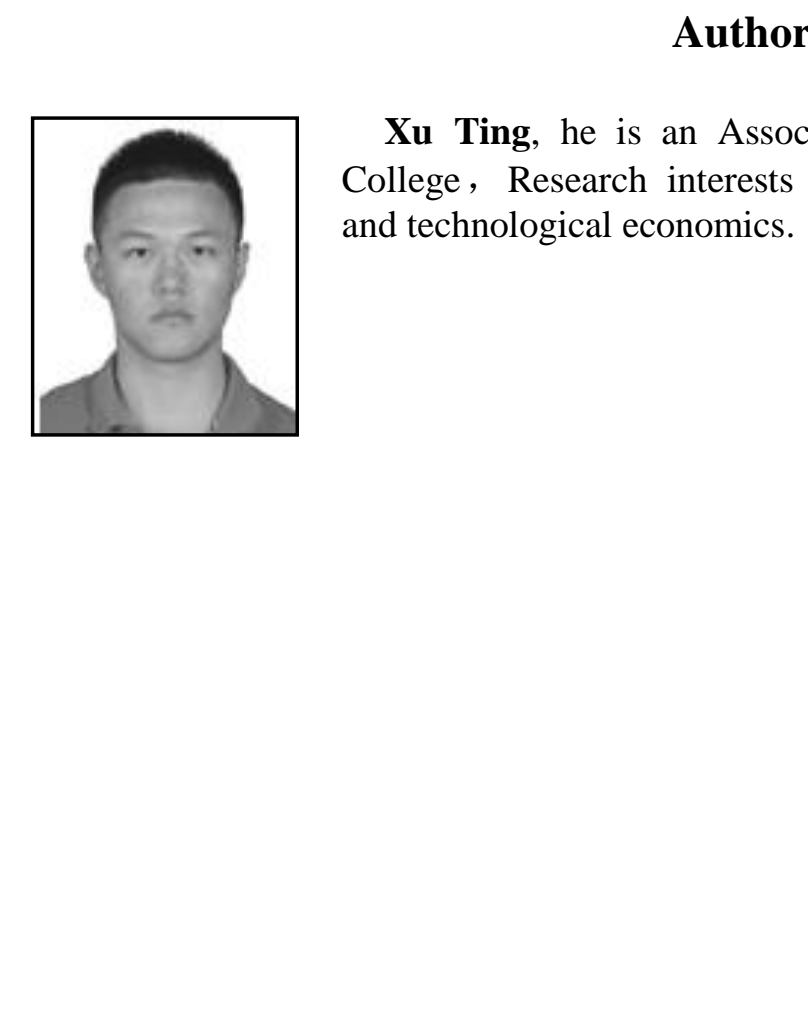


International Journal of Hybrid Information Technology

Vol. 9, No.10 (2016)

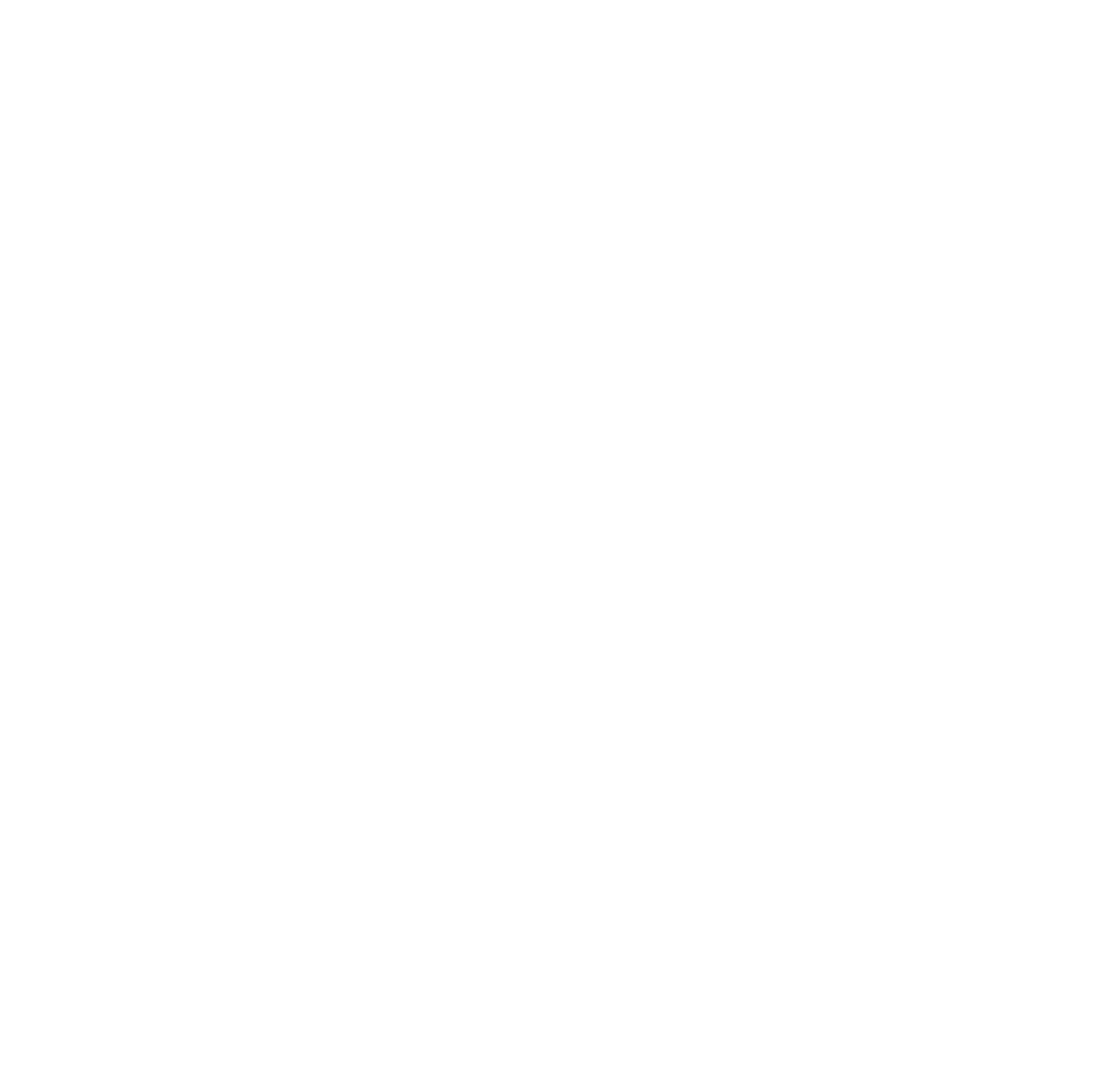

